\title{
Elliptic hypergeometric integrals and 't Hooft anomaly matching conditions
}

\author{
V.P. Spiridonov ${ }^{a}$ and G.S. Vartanov ${ }^{b}$ \\ ${ }^{a}$ Bogoliubov Laboratory of Theoretical Physics, \\ JINR, Dubna, Moscow Region 141980, Russia \\ ${ }^{b}$ DESY Theory, \\ Notkestrasse 85, 22603 Hamburg, Germany \\ E-mail: spiridon@theor.jinr.ru, grigory.vartanov@desy.de
}

ABSTRACT: Elliptic hypergeometric integrals describe superconformal indices of $4 d$ supersymmetric field theories. We show that all 't Hooft anomaly matching conditions for Seiberg dual theories can be derived from $\operatorname{SL}(3, \mathbb{Z})$-modular transformation properties of the kernels of dual indices.

Keywords: Supersymmetry and Duality, Anomalies in Field and String Theories

ARXiv EPRINT: 1203.5677

To the memory of F.A. Dolan 


\section{Contents}

1 Superconformal index 1

2 The modified elliptic gamma function $\quad 4$

3 The total ellipticity condition and anomaly matchings $\quad 6$

$4 \quad \operatorname{SL}(3, \mathbb{Z})$-modularity and anomalies $\quad 12$

5 Total ellipticity and modularity. The general case 16

$\begin{array}{llr}6 & \text { Conclusion } & 18\end{array}$

\section{Superconformal index}

In a remarkable paper [1] Dolan and Osborn have recognized the fact that superconformal indices (SCIs) of $4 d$ supersymmetric gauge theories [2-4] are expressed in terms of elliptic hypergeometric integrals (EHIs) [5,6] (see [7] for a review). This observation provides currently the most rigorous mathematical confirmation of $\mathcal{N}=1$ Seiberg electric-magnetic duality [8] through the equality of dual indices. In a sequel of papers [9-15] we have systematically studied this interrelation between SCIs and EHIs and described many new $\mathcal{N}=1$ physical dualities and conjectured many new identities for EHIs. Supersymmetric field theories on curved backgrounds and corresponding indices modeling SCIs have been studied in [16-18]. The theory of EHIs was applied also to a description of the $S$-duality conjecture for $\mathcal{N}=2,4$ extended supersymmetric field theories [10, 12, 19-22]. Several modifications of SCIs have been considered recently such as the inclusion of charge conjugation [23], indices on lens spaces [24], inclusion of surface operators [25] or line operators [26, 27]. In [14] it was shown that SCIs of $4 d$ theories describe partition functions of some novel integrable models of $2 d$ spins systems where the Seiberg duality plays the role of Kramers-Wannier duality transformations.

By definition SCIs count BPS states protected by one supersymmetry which can not be combined to form long multiplets. The $\mathcal{N}=1$ superconformal algebra of $\mathrm{SU}(2,2 \mid 1)$ spacetime symmetry group is generated by $J_{i}, \bar{J}_{i}$ (Lorentz rotations), $P_{\mu}$ (translations), $K_{\mu}$, (special conformal transformations), $H$ (dilatations) and $R\left(\mathrm{U}(1)_{R}\right.$-rotations). In addition to the bosonic generators there are supercharges $Q_{\alpha}, \bar{Q}_{\dot{\alpha}}$ and their superconformal partners $S_{\alpha}, \bar{S}_{\dot{\alpha}}$. Distinguishing a pair of supercharges, say, $Q=\bar{Q}_{1}$ and $Q^{\dagger}=-\bar{S}_{1}$, one has

$$
\left\{Q, Q^{\dagger}\right\}=2 \mathcal{H}, \quad Q^{2}=\left(Q^{\dagger}\right)^{2}=0, \quad \mathcal{H}=H-2 \bar{J}_{3}-3 R / 2 .
$$




\begin{tabular}{|c|c|c|c|c|c|}
\hline & $\mathrm{SU}\left(N_{c}\right)$ & $\mathrm{SU}\left(N_{f}\right)_{l}$ & $\mathrm{SU}\left(N_{f}\right)_{r}$ & $\mathrm{U}(1)_{B}$ & $\mathrm{U}(1)_{R}$ \\
\hline$Q$ & $f$ & $f$ & 1 & 1 & $\frac{N_{f}-N_{c}}{N_{f}}$ \\
$\widetilde{Q}$ & $\bar{f}$ & 1 & $\bar{f}$ & -1 & $\frac{N_{f}-N_{c}}{N_{f}}$ \\
$V$ & $\operatorname{adj}$ & 1 & 1 & 0 & 1 \\
\hline
\end{tabular}

Table 1. Matter content of the electric theory.

The superconformal index is defined now by the trace

$$
I\left(p, q, f_{k}\right)=\operatorname{Tr}\left((-1)^{\mathcal{F}} p^{\mathcal{R} / 2+J_{3}} q^{\mathcal{R} / 2-J_{3}} e^{\sum_{k} f_{k} F_{k}} e^{-\beta \mathcal{H}}\right), \quad \mathcal{R}=R+2 \bar{J}_{3},
$$

where $\mathcal{F}$ is the fermion number operator. Chemical potentials $f_{k}$ are the group parameters of the flavor symmetry group with the maximal torus generators $F_{k}$. The variables $p$ and $q$ are fugacities (group parameters) for the operators $\mathcal{R} / 2 \pm J_{3}$ commuting with $Q$ and $Q^{\dagger}$. Only zero modes of $\mathcal{H}$ contribute to the trace because relation (1.1) is preserved by the operators used in (1.2).

An explicit computation of SCIs for $\mathcal{N}=1$ theories results in the prescription $[1,4]$ according to which one should first compute the trace in (1.2) over the single particle states

$$
\begin{aligned}
\operatorname{ind}(p, q, \underline{z}, \underline{y})= & \frac{2 p q-p-q}{(1-p)(1-q)} \chi_{\mathrm{adj}, G}(\underline{z}) \\
& +\sum_{j} \frac{(p q)^{R_{j} / 2} \chi_{R_{F}, j}(\underline{y}) \chi_{R_{G}, j}(\underline{z})-(p q)^{1-R_{j} / 2} \chi_{\bar{R}_{F}, j}(\underline{y}) \chi_{\bar{R}_{G}, j}(\underline{z})}{(1-p)(1-q)},
\end{aligned}
$$

where the first term describes the contribution of gauge superfields lying in the adjoint representation of the gauge group $G_{c}$. The sum over $j$ corresponds to the contribution of chiral matter superfields $\Phi_{j}$ transforming as the gauge group representations $R_{G, j}$ and flavor symmetry group representations $R_{F, j}$. The functions $\chi_{\text {adj }}(\underline{z}), \chi_{R_{F}, j}(\underline{y})$ and $\chi_{R_{G}, j}(\underline{z})$ are the corresponding characters. The exponents $R_{j}$ are the field $R$-charges. To obtain the full SCI, this single particle states index is inserted into the "plethystic" exponential with the subsequent averaging over the gauge group leading to the matrix integral

$$
I(p, q, \underline{y})=\int_{G_{c}} d \mu(\underline{z}) \exp \left(\sum_{n=1}^{\infty} \frac{1}{n} \operatorname{ind}\left(p^{n}, q^{n}, \underline{z}^{n}, \underline{y}^{n}\right)\right),
$$

where $d \mu(\underline{z})$ is the $G_{c^{-}}$invariant measure.

Let us take the initial Seiberg duality for SQCD [8] and consider it in detail. Namely, take a $4 d \mathcal{N}=1 \mathrm{SYM}$ theory with $G_{c}=\mathrm{SU}\left(N_{c}\right)$ gauge group and $\mathrm{SU}\left(N_{f}\right)_{l} \times \mathrm{SU}\left(N_{f}\right)_{r} \times$ $\mathrm{U}(1)_{B}$ flavor symmetry group. The original (electric) theory has $N_{f}$ left and $N_{f}$ right quarks $Q$ and $\widetilde{Q}$ lying in the fundamental and antifundamental representation of $\operatorname{SU}\left(N_{c}\right)$ and having +1 and -1 baryonic charges and the $R$-charge $R=\left(N_{f}-N_{c}\right) / N_{f}$ (this is the $R$-charge for the scalar component, the $R$-charge of the fermion component is $R-1)$. The field content of the described theory is summarized in table 1. 


\begin{tabular}{|c|c|c|c|c|c|}
\hline & $\mathrm{SU}\left(\widetilde{N}_{c}\right)$ & $\mathrm{SU}\left(N_{f}\right)_{l}$ & $\mathrm{SU}\left(N_{f}\right)_{r}$ & $\mathrm{U}(1)_{B}$ & $\mathrm{U}(1)_{R}$ \\
\hline$M$ & 1 & $f$ & $\bar{f}$ & 0 & $2 \frac{\widetilde{N}_{c}}{N_{f}}$ \\
$q$ & $f$ & $\bar{f}$ & 1 & $\frac{N_{c}}{\widetilde{N}_{c}}$ & $\frac{N_{c}}{N_{f}}$ \\
$\widetilde{q}$ & $\bar{f}$ & 1 & $f$ & $-\frac{N_{c}}{\widetilde{N}_{c}}$ & $\frac{N_{c}}{N_{f}}$ \\
$\widetilde{V}$ & $\operatorname{adj}$ & 1 & 1 & 0 & 1 \\
\hline
\end{tabular}

Table 2. Matter content of the magnetic theory.

Corresponding SCI is given by the following elliptic hypergeometric integral [1]

$$
I_{E}=\kappa_{N_{c}} \int_{\mathbb{T}^{N_{c}-1}} \frac{\prod_{i=1}^{N_{f}} \prod_{j=1}^{N_{c}} \Gamma\left(s_{i} z_{j}, t_{i}^{-1} z_{j}^{-1} ; p, q\right)}{\prod_{1 \leq i<j \leq N_{c}} \Gamma\left(z_{i} z_{j}^{-1}, z_{i}^{-1} z_{j} ; p, q\right)} \prod_{j=1}^{N_{c}-1} \frac{d z_{j}}{2 \pi \mathrm{i} z_{j}},
$$

where $\mathbb{T}$ denotes the unit circle with positive orientation, $\prod_{j=1}^{N_{c}} z_{j}=1,\left|s_{i}\right|,\left|t_{i}^{-1}\right|<1$, and the balancing condition reads $S T^{-1}=(p q)^{N_{f}-N_{c}}$ with $S=\prod_{i=1}^{N_{f}} s_{i}, T=\prod_{i=1}^{N_{f}} t_{i}$. Here we introduced the parameters $s_{i}$ and $t_{i}$ as

$$
s_{i}=(p q)^{R / 2} v x_{i}, \quad t_{i}=(p q)^{-R / 2} v y_{i},
$$

where $v, x_{i}$ and $y_{i}$ are fugacities for $\mathrm{U}(1)_{B}, \mathrm{SU}\left(N_{f}\right)_{l}$ and $\mathrm{SU}\left(N_{f}\right)_{r}$ groups, respectively, with the constraints $\prod_{i=1}^{N_{f}} x_{i}=\prod_{i=1}^{N_{f}} y_{i}=1$, and

$$
\kappa_{N_{c}}=\frac{(p ; p)_{\infty}^{N_{c}-1}(q ; q)_{\infty}^{N_{c}-1}}{N_{c} !}, \quad(a ; q)_{\infty}=\prod_{k=0}^{\infty}\left(1-a q^{k}\right) .
$$

We use also conventions

$$
\Gamma(a, b ; p, q):=\Gamma(a ; p, q) \Gamma(b ; p, q), \quad \Gamma\left(a z^{ \pm 1} ; p, q\right):=\Gamma(a z ; p, q) \Gamma\left(a z^{-1} ; p, q\right),
$$

where

$$
\Gamma(z ; p, q)=\prod_{i, j=0}^{\infty} \frac{1-z^{-1} p^{i+1} q^{j+1}}{1-z p^{i} q^{j}}, \quad|p|,|q|<1,
$$

is the (standard) elliptic gamma function.

According to [8] the dual (magnetic) theory is described by a $4 d \mathcal{N}=1 \mathrm{SYM}$ theory with the gauge group $\widetilde{G}_{c}=\mathrm{SU}\left(\widetilde{N}_{c}\right), \widetilde{N}_{c}=N_{f}-N_{c}$, sharing the same flavor symmetry. Here one has dual quarks $q$ and $\widetilde{q}$ lying in the fundamental and antifundamental representation of $\widetilde{G}_{c}$ with $\mathrm{U}(1)_{B}$-charges $N_{c} /\left(N_{f}-N_{c}\right)$ and $-N_{c} /\left(N_{f}-N_{c}\right)$ and the $R$-charges $N_{c} / N_{f}$. Additionally, there are mesons - singlets of $\widetilde{G}_{c}$ lying in the fundamental representation of $\mathrm{SU}\left(N_{f}\right)_{l}$ and antifundamental representation of $\mathrm{SU}\left(N_{f}\right)_{r}\left(M_{i}^{j}=Q_{i} \widetilde{Q}^{j}, i, j=1, \ldots, N_{f}\right)$. It is convenient to collect again all fields data in table 2 .

These two SQCD-type theories are dual to each other in their infrared fixed points when the magnetic theory has a dynamically generated superpotential [8], $W_{d y n}=M_{i}^{j} q^{i} \widetilde{q}_{j}$. 
The SCI of the magnetic theory is

$$
\begin{aligned}
I_{M}= & \kappa_{N_{\widetilde{N}_{c}}} \prod_{1 \leq i, j \leq N_{f}} \Gamma\left(s_{i} t_{j}^{-1} ; p, q\right) \\
& \times \int_{\mathbb{T}^{N_{c}-1}} \frac{\prod_{i=1}^{N_{f}} \prod_{j=1}^{\widetilde{N}_{c}} \Gamma\left(S^{1 / \widetilde{N}_{c}} s_{i}^{-1} \widetilde{z}_{j}, T^{-1 / \widetilde{N}_{c}} t_{i} \widetilde{z}_{j}^{-1} ; p, q\right)}{\prod_{1 \leq i<j \leq \widetilde{N}_{c}} \Gamma\left(\widetilde{z}_{i} \widetilde{z}_{j}^{-1}, \widetilde{z}_{i}^{-1} \widetilde{z}_{j} ; p, q\right)} \prod_{j=1}^{\widetilde{N}_{c}-1} \frac{d \widetilde{z}_{j}}{2 \pi \mathrm{i} \widetilde{z}_{j}}
\end{aligned}
$$

where $\prod_{j=1}^{\widetilde{N}_{c}} \widetilde{z}_{j}=1$, and $\left|S^{1 / \widetilde{N}_{c}} s_{i}^{-1}\right|,\left|T^{-1 / \widetilde{N}_{c}} t_{i}\right|<1$. As discovered by Dolan and Osborn [1], the equality of SCIs $I_{E}=I_{M}$ coincides with the mathematical identity initially considered for $N_{c}=2, N_{f}=3,4$ and $N_{f}=N_{c}+1$ in [5-7] and established for arbitrary parameters in [28].

In $[10,11]$ we proposed to relate known physical checks of the Seiberg duality to certain mathematical properties of EHIs:

1. 't Hooft anomaly matching conditions for dual theories [29] were conjectured to follow from the so-called total ellipticity property for elliptic hypergeometric terms [7, 30].

2. One can reduce the duality from $N_{f}$ to $N_{f}-1$ flavors by integrating out one flavor. At the level of SCIs this can be realized by the restriction $s_{f} t_{f}^{-1}=p q$ for fugacities of the flavor $f$ one wants to integrate out.

3. Matching of the moduli spaces and gauge invariant operators should correspond to the equality of coefficients in the series expansions of SCIs having a topological meaning.

The main purpose of this paper is to analyze in detail the first point of this list. amely, we show that our original conjecture (1) is not completely true, i.e. the total ellipticity condition is not sufficient to match all anomalies. Instead, all continuous current anomalies match as a consequence of the nontrivial $\mathrm{SL}(3, \mathbb{Z})$-modular group properties of the kernels of elliptic hypergeometric integrals describing indices. The importance of this modular group for dualities was announced by us in [31].

\section{The modified elliptic gamma function}

We start from describing the modified elliptic gamma function [6] playing a key role in our considerations. Function (1.6) satisfies the following equations

$$
\Gamma(q z ; p, q)=\theta(z ; p) \Gamma(z ; p, q), \quad \Gamma(p z ; p, q)=\theta(z ; q) \Gamma(z ; p, q),
$$

where $\theta(z ; p)$ is a theta-function

$$
\theta(z ; p)=(z ; p)_{\infty}\left(p z^{-1} ; p\right)_{\infty} .
$$

This (shortened) theta function satisfies symmetry properties

$$
\theta(p z ; p)=\theta\left(z^{-1} ; p\right)=-z \theta(z ; p)
$$


and for any $k \in \mathbb{Z}$

$$
\theta\left(p^{k} z ; p\right)=(-z)^{-k} p^{-k(k-1) / 2} \theta(z ; p) .
$$

Equations (2.1) necessarily demand that $|p|,|q|<1$, and for $p^{n} \neq q^{m}, n, m \in \mathbb{Z}$, they define $\Gamma(z ; p, q)$ uniquely as a meromorphic function of $z \in \mathbb{C}^{*}$ with the normalization $\Gamma(\sqrt{p q} ; p, q)=1$.

Let us take three complex variables $\omega_{1}, \omega_{2}, \omega_{3}$ and define the bases

$$
p=e^{2 \pi \mathrm{i} \omega_{3} / \omega_{2}}, \quad q=e^{2 \pi \mathrm{i} \omega_{1} / \omega_{2}}, \quad r=e^{2 \pi \mathrm{i} \omega_{3} / \omega_{1}}
$$

together with their particular modular transformed partners

$$
\widetilde{p}=e^{-2 \pi \mathrm{i} \omega_{2} / \omega_{3}}, \quad \widetilde{q}=e^{-2 \pi \mathrm{i} \omega_{2} / \omega_{1}}, \quad \widetilde{r}=e^{-2 \pi \mathrm{i} \omega_{1} / \omega_{3}} .
$$

Remind now that the elliptic gamma function is originally related to one finite difference equation

$$
f\left(u+\omega_{1}\right)=\theta\left(e^{2 \pi \mathrm{i} u / \omega_{2}} ; p\right) f(u), u \in \mathbb{C} .
$$

It coincides with the first equation above for $\Gamma(z ; p, q)$ with $z=e^{2 \pi \mathrm{i} u / \omega_{2}}$, but it assumes only one constraint for bases, $|p|<1$. There exist other nontrivial solutions to (2.5) which do not require $|q|<1$. Namely, consider equation (2.5) together with two additional equations

$$
f\left(u+\omega_{2}\right)=\theta\left(e^{2 \pi \mathrm{i} u / \omega_{1}} ; r\right) f(u), \quad f\left(u+\omega_{3}\right)=e^{-\pi \mathrm{i} B_{2,2}(u ; \omega)} f(u),
$$

where $B_{2,2}(u ; \omega)$ is a second order Bernoulli polynomial

$$
B_{2,2}(u ; \omega)=\frac{u^{2}}{\omega_{1} \omega_{2}}-\frac{u}{\omega_{1}}-\frac{u}{\omega_{2}}+\frac{\omega_{1}}{6 \omega_{2}}+\frac{\omega_{2}}{6 \omega_{1}}+\frac{1}{2} .
$$

Then for incommensurate $\omega_{j}$ 's the modified elliptic gamma function [6]

$$
\mathcal{G}(u ; \omega)=\Gamma\left(e^{2 \pi \mathrm{i} u / \omega_{2}} ; p, q\right) \Gamma\left(r e^{-2 \pi \mathrm{i} u / \omega_{1}} ; \widetilde{q}, r\right)
$$

defines the unique meromorphic solution of these three equations satisfying the normalization condition $\mathcal{G}\left(\sum_{i=1}^{3} \omega_{i} / 2 ; \omega\right)=1$. This is a meromorphic function of $u$ even for $\omega_{1} / \omega_{2}>0$, when $|q|=1$, which is easily seen from its another representation

$$
\mathcal{G}(u ; \omega)=e^{-\frac{\pi \mathrm{i}}{3} B_{3,3}(u ; \omega)} \Gamma\left(e^{-2 \pi \mathrm{i} u / \omega_{3}} ; \widetilde{r}, \widetilde{p}\right),
$$

where $B_{3,3}$ is a Bernoulli polynomial of the third order

$$
B_{3,3}(u ; \omega)=\frac{1}{\omega_{1} \omega_{2} \omega_{3}}\left(u-\frac{1}{2} \sum_{k=1}^{3} \omega_{k}\right)\left(\left(u-\frac{1}{2} \sum_{k=1}^{3} \omega_{k}\right)^{2}-\frac{1}{4} \sum_{k=1}^{3} \omega_{k}^{2}\right) .
$$

Multiple Bernoulli polynomials are defined in the theory of Barnes multiple zeta-function [7] from the following expansion

$$
\frac{x^{m} e^{x u}}{\prod_{k=1}^{m}\left(e^{\omega_{k} x}-1\right)}=\sum_{n=0}^{\infty} B_{m, n}\left(u ; \omega_{1}, \ldots, \omega_{m}\right) \frac{x^{n}}{n !} .
$$




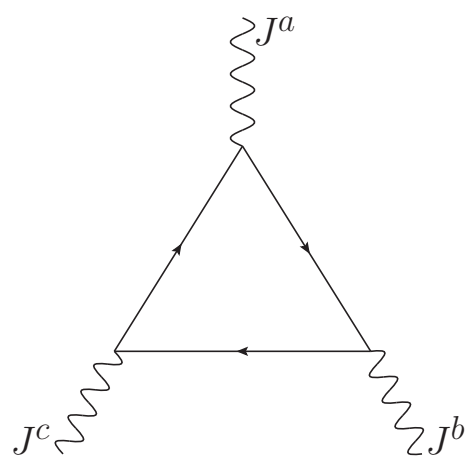

Figure 1. A one-loop triangle diagram describing anomalies.

The equality of expressions (2.7) and (2.8) follows from the Jacobi theorem on absence of nontrivial triply periodic functions, since both expressions satisfy three equations and the normalization condition indicated above. This equality represents a modular transformation law from the $\mathrm{SL}(3, \mathbb{Z})$-group [32]. We stress that all three bases $p, q, r$ are involved into the description of $\mathcal{G}(u ; \omega)$. The modified elliptic hypergeometric integrals built from the modified elliptic gamma functions [33] and the Bernoulli polynomial (2.9) will play the major role in our analysis below.

We shall use also the well known modular transformation properties of the theta function

$$
\theta\left(e^{-2 \pi \mathrm{i} u / \omega_{1}} ; e^{-2 \pi \mathrm{i} \omega_{2} / \omega_{1}}\right)=e^{\pi \mathrm{i} B_{2,2}\left(u ; \omega_{1}, \omega_{2}\right)} \theta\left(e^{2 \pi \mathrm{i} u / \omega_{2}} ; e^{2 \pi \mathrm{i} \omega_{1} / \omega_{2}}\right)
$$

and of the Dedekind eta-function

$$
e^{-\frac{\pi \mathrm{i}}{12 \tau}}\left(e^{-2 \pi \mathrm{i} / \tau} ; e^{-2 \pi \mathrm{i} / \tau}\right)_{\infty}=(-\mathrm{i} \tau)^{1 / 2} e^{\frac{\pi \mathrm{i} \tau}{12}}\left(e^{2 \pi \mathrm{i} \tau} ; e^{2 \pi \mathrm{i} \tau}\right)_{\infty},
$$

where $\sqrt{-\mathrm{i}}=e^{-\pi \mathrm{i} / 4}$.

\section{$3 \quad$ The total ellipticity condition and anomaly matchings}

Anomalies. We would like to remind basic facts about the anomalies and 't Hooft anomaly matching conditions. All continuous symmetry anomalies are obtained from the one-loop triangular diagrams presented in figure 1 where loop lines contain all possible fermions and external lines are either global symmetry currents, gauge currents or graviton currents (there are two such diagrams: the second one is obtained from figure 1 by reversing the fermion current). When all external lines describe gauge currents one gets the local gauge invariance anomalies which should cancel to have a consistent theory.

Calculation of the triangle diagram is the same for all anomalies, the only difference being described by the group-theoretical factor. The triangle diagram $\left\langle j_{G}^{a, \mu} j_{G}^{b, \nu} j_{G}^{c, \lambda}\right\rangle$, where $j_{G}^{a, \mu}=\bar{\psi} \gamma^{\mu} t^{a} \psi$ with $\psi$ being the fermion component of a chiral superfield or the gluino, is proportional to $\mathcal{A}(\mathcal{R}) d^{a b c}=\operatorname{Tr}\left[t^{a}\left\{t^{b}, t^{c}\right\}\right]$ (the trace is taken over $G_{c^{-}}$group matrices in 
some representation $\mathcal{R}$ ). The total anomaly is proportional to the sum over all fermions $\sum_{\text {fermions }} \operatorname{Tr}\left[t^{a}\left\{t^{b}, t^{c}\right\}\right]$. In our electric theory the explicit calculation boils to the equality

$$
\text { (1) } N_{f}+(-1) N_{f}+0=0 \text {, }
$$

since the triple Casimir invariant of $G_{c}=\mathrm{SU}\left(N_{c}\right)$ for the fundamental representation is $\mathcal{A}(f)=1$, for the antifundamental one $\mathcal{A}(\bar{f})=-1$, and for the adjoint representation $\mathcal{A}(\operatorname{adj})=0$.

In the definition of SCIs (1.2) it is assumed that all operators entering it represent exact physical symmetries. This means that the corresponding currents are not anomalous $\left\langle j_{G}^{a, \mu} j_{G}^{b, \nu} j_{1}^{\lambda}\right\rangle=0$, where $j_{1}^{\lambda}$ is $\mathrm{U}(1)_{R}$ or any flavor symmetry current (in the infrared fixed point the $R$-charge should be conserved similar to the energy-momentum). In the $\mathrm{U}(1)_{R^{-}}$ case this anomaly coefficient is proportional to $R \operatorname{Tr}\left\{t^{a} t^{b}\right\}=R T(\mathcal{R}) \delta^{a b}$, where $R$ is the $R$-charge. In the electric theory one has

$$
(R-1) 2 N_{f} \frac{1}{2}+N_{c}=0,
$$

since the Casimir operators of $G_{c}=\mathrm{SU}\left(N_{c}\right)$ for fundamental, antifundamental, and adjoint representations are $T(f)=T(\bar{f})=1 / 2$, and $T(\operatorname{adj})=N_{c}$, respectively. Here $R-1$ is the $R$-charge of chiral quarks and the $R$-charge of gluinos is equal to 1 . As a result, one fixes the $R$-charge of chiral superfields, $R=\left(N_{f}-N_{c}\right) / N_{f}$. Similarly, gauge invariance yields $\left\langle j_{G}^{a, \mu} j_{1}^{\nu} j_{2}^{\lambda}\right\rangle=0$ for any conserved global symmetry current $j_{1}^{\nu}$ and $j_{2}^{\lambda}$.

As to the anomalies associated only with global symmetry groups - they are not obliged to vanish. As argued by 't Hooft [29], for any electric-magnetic duality (including the Seiberg $\mathcal{N}=1$ duality) the coefficients of admissible triangle anomalies should match in dual theories. For example, in the Seiberg case $\mathrm{SU}\left(N_{f}\right)_{l}^{3}$-anomaly is described by $\left\langle j_{\mathrm{SU}\left(N_{f}\right)_{l}}^{a, \mu} j_{\mathrm{SU}\left(N_{f}\right)_{l}}^{b, \nu} j_{\mathrm{SU}\left(N_{f}\right)_{l}}^{c, \lambda}\right\rangle$ with $j_{\mathrm{SU}\left(N_{f}\right)_{l}}^{a, \mu}=\bar{\psi} \gamma^{\mu} t^{a} \psi$, where $t^{a}$ is the $\mathrm{SU}\left(N_{f}\right)_{l}$ fundamental representation matrix. For the electric theory the anomaly coefficient comes only from one field and equals to $(1) N_{c}$, while in the magnetic side one has two different contributions (from dual quarks $q$ and mesons $M$ ) which yield the coefficient $(-1)\left(N_{f}-N_{c}\right)+N_{f}=N_{c}$ confirming one of the 't Hooft anomaly matching conditions.

Being a quantitative check, the 't Hooft anomaly matching conditions provide an extremely powerful tool for checking $4 d \mathcal{N}=1$ dualities. Still, one should be careful with these conditions, see e.g. [34], where examples of misleading anomaly matching conditions were found. Namely, there are $\mathcal{N}=1 \mathrm{SYM}$ theories with equal anomaly coefficients, but the deformation by mass parameters argument shows that these theories are not dual to each other. From the SCI point of view this fact is reflected in the difference of analytical structure of SCIs [13].

We consider explicitly only the original Seiberg duality assuming that other dualities can be treated in a similar way. For a further comparison we give a full list of corresponding 
nontrivial anomaly coefficients:

$$
\begin{aligned}
& \mathrm{SU}\left(N_{f}\right)_{l, r}^{3}: N_{c}, \quad \mathrm{SU}\left(N_{f}\right)_{l, r}^{2} \mathrm{U}(1)_{B}: \frac{N_{c}}{2}, \\
& \mathrm{SU}\left(N_{f}\right)_{l, r}^{2} \mathrm{U}(1)_{R}:(R-1) N_{c} \frac{1}{2}=-\frac{N_{c}^{2}}{2 N_{f}}, \\
& \mathrm{U}(1)_{B}^{2} \mathrm{U}(1)_{R}: \quad(R-1) 2 N_{f} N_{c}=-2 N_{c}^{2}, \\
& \mathrm{U}(1)_{R}: \quad(R-1) 2 N_{f} N_{c}+N_{c}^{2}-1=-N_{c}^{2}-1, \\
& \mathrm{U}(1)_{R}^{3}: \quad(R-1)^{3} 2 N_{f} N_{c}+N_{c}^{2}-1=-2 \frac{N_{c}^{4}}{N_{f}^{2}}+N_{c}^{2}-1,
\end{aligned}
$$

where $R=\left(N_{f}-N_{c}\right) / N_{f}$. Note that in the case of $\mathrm{U}(1)_{R^{-}}$current anomaly the triangle diagrams involve two gravitational currents.

The total ellipticity condition. The notion of total ellipticity was introduced first for elliptic hypergeometric series [7] which we skip. An elliptic function is called totally elliptic if it is doubly periodic in all continuous variables used to parametrize its divisor space of maximal possible dimension. A meromorphic function is called the elliptic hypergeometric term if it satisfies a homogeneous linear difference equation in one of the variables with the coefficient which is elliptic in this variable. Elliptic hypergeometric term is called totally elliptic if it satisfies such equations in each variable with the coefficients which are totally elliptic functions [30]. It is believed that one can associate a supersymmetric duality with each nontrivial totally elliptic hypergeometric term formed as the ratio of the kernels of two differently looking, but equal integrals [10].

In [30] the total ellipticity condition for the equality of integrals of interest (1.4) and (1.7) has been checked. We partially repeat here corresponding calculations. First, we change variables $\underline{\widetilde{z}}$ in (1.7) to $\underline{\tilde{z}}=S^{-1 / \widetilde{N}_{c}} \underline{w}$. Then the equality of integrals (1.4) and (1.7) is rewritten in the following way

$$
\begin{aligned}
\int_{\mathbb{T}^{N_{c}-1}} \Delta_{E}(\underline{z}, \underline{t}, \underline{s}) \prod_{j=1}^{N_{c}-1} \frac{d z_{j}}{2 \pi \mathrm{i} z_{j}} & =\int_{\left(S^{\left.1 / \widetilde{N}_{c} \mathbb{T}\right)^{N_{c}-1}}\right.} \Delta_{M}(\underline{w}, \underline{t}, \underline{s}) \prod_{j=1}^{\widetilde{N}_{c}-1} \frac{d w_{j}}{2 \pi \mathrm{i} w_{j}}, \\
\Delta_{E}(\underline{z}, \underline{s}, \underline{t}) & =\kappa_{N_{c}} \frac{\prod_{i=1}^{N_{f}} \prod_{j=1}^{N_{c}} \Gamma\left(s_{i} z_{j}, t_{i}^{-1} z_{j}^{-1} ; p, q\right)}{\prod_{1 \leq i<j \leq N_{c}} \Gamma\left(z_{i} z_{j}^{-1}, z_{i}^{-1} z_{j} ; p, q\right)}, \\
\Delta_{M}(\underline{w}, \underline{s}, \underline{t}) & =\kappa_{\widetilde{N}_{c}} \prod_{i, j=1}^{N_{f}} \Gamma\left(s_{i} t_{j}^{-1} ; p, q\right) \frac{\prod_{i=1}^{N_{f}} \prod_{j=1}^{\widetilde{N}_{c}} \Gamma\left(s_{i}^{-1} w_{j}, p q t_{i} w_{j}^{-1} ; p, q\right)}{\prod_{1 \leq i<j \leq \widetilde{N}_{c}} \Gamma\left(w_{i} w_{j}^{-1}, w_{i}^{-1} w_{j} ; p, q\right)},
\end{aligned}
$$

with $\prod_{i=1}^{N_{c}} z_{i}=1$ and $\prod_{i=1}^{\widetilde{N}_{c}} w_{i}=S$. Consider the function

$$
\rho(\underline{z}, \underline{w}, \underline{s}, \underline{t} ; p, q)=\frac{\Delta_{E}(\underline{z}, \underline{s}, \underline{t})}{\Delta_{M}(\underline{w}, \underline{s}, \underline{t})}
$$


and the ratios, called $q$-certificates,

$$
h^{g}(\underline{z}, \underline{w}, \underline{s}, \underline{t}, q ; p)=\frac{\rho(\ldots, q g, \ldots ; p, q)}{\rho(\ldots, g, \ldots ; p, q)}, \quad g \in\{\underline{z}, \underline{w}, \underline{s}, \underline{t}\} .
$$

The total ellipticity condition for $\rho(\underline{z}, \underline{w}, \underline{s}, \underline{t} ; p, q)$ is then formulated as the requirement for all $h^{g}$-functions to be $p$-elliptic in all variables $\{\underline{z}, \underline{w}, \underline{s}, \underline{t}, q\}$, i.e. they should not change under the $p$-shifts $z_{i} \rightarrow p^{\alpha_{i}} z_{i}, w_{i} \rightarrow p^{\beta_{i}} w_{i}, s_{k} \rightarrow p^{\gamma_{k}} s_{k}, t_{k} \rightarrow p^{\mu_{k}} t_{k}, q \rightarrow p^{\nu} q, \alpha_{i}, \ldots, \nu \in \mathbb{Z}$, provided all the additional constraints for fugacities are satisfied. We remind that according to the original definition given in [6], a contour integral with integration variables $z_{i}$ is called the elliptic hypergeometric integral if $h^{z_{i}}$-certificates built from its kernel are $p$-elliptic in all $\underline{z}$, which is a much weaker condition.

Let $m_{j}^{(a)} \in \mathbb{Z}, j=1, \ldots, n, a=1, \ldots, K$, and $\epsilon\left(m^{(a)}\right)=\epsilon\left(m_{1}^{(a)}, \ldots, m_{n}^{(a)}\right)$ are arbitrary $\mathbb{Z}^{n} \rightarrow \mathbb{Z}$ maps with finite support and $r_{-} \in \mathbb{Z}$. Define a meromorphic function of free variables $x_{i} \in \mathbb{C}^{*}, i=1, \ldots, n$,

$$
\Delta\left(x_{1}, \ldots, x_{n} ; p, q\right)=(p ; p)_{\infty}^{r_{-}}(q ; q)_{\infty}^{r_{-}} \prod_{a=1}^{K} \Gamma\left(x_{1}^{m_{1}^{(a)}} x_{2}^{m_{2}^{(a)}} \ldots x_{n}^{m_{n}^{(a)}} ; p, q\right)^{\epsilon\left(m^{(a)}\right)} .
$$

The following theorem was presented in [30].

Theorem 1 (Rains, Spiridonov, 2004) Suppose $\Delta(x ; p, q)$ is a totally elliptic hypergeometric term, i.e. all its q-certificates

$$
h_{i}(x, q ; p)=\frac{\Delta\left(\ldots, q x_{i}, \ldots ; p, q\right)}{\Delta\left(x_{1}, \ldots, x_{n} ; p, q\right)}=\prod_{a=1}^{K} \prod_{l=0}^{m_{i}^{(a)}-1} \theta\left(q^{l} \prod_{k=1}^{n} x_{k}^{m_{k}^{(a)}} ; p\right)^{\epsilon\left(m^{(a)}\right)}
$$

are $p$-elliptic functions of $q$ and $x_{1}, \ldots, x_{n}$. Then these $q$-certificates are also modular invariant.

The statements of the theorem are guaranteed because of the following diophantine equations

$$
\begin{aligned}
\sum_{a=1}^{K} \epsilon\left(m^{(a)}\right) m_{i}^{(a)} m_{j}^{(a)} m_{k}^{(a)} & =0, \\
\sum_{a=1}^{K} \epsilon\left(m^{(a)}\right) m_{i}^{(a)} m_{j}^{(a)} & =0, \\
\sum_{a=1}^{K} \epsilon\left(m^{(a)}\right) m_{i}^{(a)} & =0 .
\end{aligned}
$$

The proof is elementary. The demand $h_{i}\left(\ldots p x_{j} \ldots, q ; p\right)=h_{i}(x, q ; p)$ leads to equations (3.10), (3.11). Equation (3.12) emerges as a consequence of the restriction $h_{i}(x, p q ; p)=h_{i}(x, q ; p)$. The theorem statement follows after application to each theta function in $h_{i}$ the modular transformation (2.10) and use of equations (3.10)-(3.12). 
In the context of SCIs variables $x_{i}$ represent combinations of fugacities of symmetry groups, $\Delta$-function is the ratio of kernels of dual indices, and $r_{-}$is the difference of ranks of the electric and magnetic gauge groups,

$$
r_{-}=r_{e}-r_{m}, \quad r_{e}=\operatorname{rank} G_{c}, \quad r_{m}=\operatorname{rank} \widetilde{G}_{c} .
$$

During the checks of the total ellipticity condition for known dualities in [10] we have noticed that some phases of the quasiperiodicity factors emerging from $p$-shifts for contributions coming from electric (or magnetic) theories coincide with the anomaly coefficients. This observation allowed us to conjecture that the total ellipticity condition guarantees 't Hooft anomaly matchings. As will be shown below this is not the case and the RainsSpiridonov equations (3.10)-(3.12) do not describe a complete set of anomaly matchings of the Seiberg-like dual theories.

It is necessary to verify that the elliptic hypergeometric term (3.7) belongs to the class (3.9), which is not evident from its explicit form we have given. In order to see this one should take definitions (3.5) and (3.6), replace there $t_{i} \rightarrow(p q)^{-1} t_{i}$ for $i=1, \ldots, N_{f}-N_{c}$ (to remove $p q$ from the balancing condition), and apply the reflection formula $\Gamma(p q z ; p, q)=$ $1 / \Gamma\left(z^{-1} ; p, q\right)$ to elliptic gamma functions having the product $p q$ in their arguments.

We stress that the ansatz (3.9) does not describe all possible forms of the elliptic hypergeometric terms. In general one can have in the arguments of elliptic gamma functions the non-removable factors $(p q)^{R / 2}$ for some fractional numbers $R$ (e.g., this is so for the Kutasov-Schwimmer duality [35]) in which case the total ellipticity condition should be modified appropriately $[10,30]$.

Ellipticity of certificates for $z_{i}$ and gauge anomalies. Take the $q$-certificates for integration variables $\underline{z}$ obtained from (3.7) after rescaling $z_{i} \rightarrow q z_{i}$ for $i \neq N_{c}$ and $z_{N_{c}} \rightarrow q^{-1} z_{N_{c}}$ (i.e., we assume that $z_{N_{c}}=\prod_{i=1}^{N c-1} z_{i}^{-1}$ ) and written in terms of the initial variables (1.5):

$$
\begin{aligned}
h_{i}^{z}(\underline{z}, v, \underline{x}, \underline{y}, q)= & \frac{\left.\rho(\underline{z}, \underline{w}, \underline{s}, \underline{t} ; p, q)\right|_{z_{i} \rightarrow q z_{i}, z_{N_{c}} \rightarrow q^{-1} z_{N_{c}}}}{\rho(\underline{z}, \underline{w}, \underline{s}, \underline{t} ; p, q)} \\
= & \frac{\theta\left(q^{-2} z_{i}^{-1} z_{N_{c}}, q^{-1} z_{i}^{-1} z_{N_{c}} ; p\right)}{\theta\left(q z_{i} z_{N_{c}}^{-1}, z_{i} z_{N_{c}}^{-1} ; p\right)} \prod_{j=1, j \neq i}^{N_{c}-1} \frac{\theta\left(q^{-1} z_{i}^{-1} z_{j}, q^{-1} z_{j}^{-1} z_{N_{c}} ; p\right)}{\theta\left(z_{i} z_{j}^{-1}, z_{j} z_{N_{c}}^{-1} ; p\right)} \\
& \quad \times \prod_{k=1}^{N_{f}} \frac{\theta\left((p q)^{R / 2} v x_{k} z_{i},(p q)^{R / 2} v^{-1} y_{k}^{-1} z_{N_{c}}^{-1} ; p\right)}{\theta\left((p q)^{R / 2} v^{-1} y_{k}^{-1}\left(q z_{i}\right)^{-1},(p q)^{R / 2} v x_{k} q^{-1} z_{N_{c}} ; p\right)} .
\end{aligned}
$$

From the physical point of view, consideration of the $z_{i}$-variable certificate can be interpreted as the insertion of one gauge current $j_{G}^{i, \mu}$ into the triangle anomaly diagram. In terms of equations (3.10)-(3.12) it means that we deal with their subpart depending at least linearly on $m_{i}^{(a)}$ coming from $G_{c}$-fugacities. Since the dependence on $z_{i}$ in $\rho$ comes only from $\Delta_{E}$, the same result is obtained if we replace in (3.13) $\rho$ by the kernel of integral describing electric SCI, i.e. the properties of $h_{i}^{z}$ describe only the electric theory. Similar situation holds for $w_{i}$-variables associated only with the magnetic theory. 
It is easy to check that $h_{i}^{z}(\underline{z}, v, \underline{x}, \underline{y}, q)$ is a totally $p$-elliptic function:

$$
\begin{array}{r}
\frac{h_{i}^{z}\left(z_{1}, \ldots, p z_{i}, \ldots, p^{-1} z_{N_{c}}, v, \underline{x}, \underline{y}, q\right)}{h_{i}^{z}(\underline{z}, v, \underline{x}, \underline{y}, q)}=\frac{(p q)^{2\left(N_{f}-N_{c}\right)}}{(p q)^{2 R N_{f}} \prod_{i=1}^{N_{f}} x_{i}^{2} y_{i}^{-2}}=1, \\
\frac{h_{i}^{z}\left(z_{1}, \ldots, p z_{c}, \ldots, p^{-1} z_{N_{c}}, v, \underline{x}, \underline{y}, q\right)}{h_{i}^{z}(\underline{z}, v, \underline{x}, \underline{y}, q)}=\frac{(p q)^{N_{f}-N_{c}}}{(p q)^{R N_{f}} \prod_{i=1}^{N_{f}} x_{i} y_{i}^{-1}}=1, \\
\frac{h_{i}^{z}\left(\underline{z}, v, \ldots, p x_{b}, \ldots, p^{-1} x_{N_{f}}, \underline{y}, q\right)}{h_{i}^{z}(\underline{z}, v, \underline{x}, \underline{y}, q)}=\frac{h_{i}^{z}\left(\underline{z}, v, \underline{x}, \ldots, p y_{b}, \ldots, p^{-1} y_{N_{f}}, q\right)}{h_{i}^{z}(\underline{z}, v, \underline{x}, \underline{y}, q)}=1, \\
\frac{h_{i}^{z}(\underline{z}, p v, \underline{x}, \underline{y}, q)}{h_{i}^{z}(\underline{z}, v, \underline{x}, \underline{y}, q)}=1,
\end{array}
$$

where $c \neq i$. The most complicate looking identity is

$$
\frac{h_{i}^{z}\left(\underline{z}, p^{R / 2} v, p^{R\left(N_{f}-1\right)} x_{1}, p^{-R} x_{2}, \ldots, p^{-R} x_{N_{f}}, \underline{y}, p q\right)}{h_{i}^{z}(\underline{z}, v, \underline{x}, \underline{y}, q)}=1,
$$

and its obvious partners obtained by permutation of $x_{j}$ together with similar equations involving $y_{j}$-variables. In terms of the variables $s_{j}, t_{j}$ this symmetry looks more compact: one has the transformations $s_{a} \rightarrow p^{N_{f}-N_{c}} s_{a}$ (or $t_{a} \rightarrow p^{N_{c}-N_{f}} t_{a}$ ) for one fixed value of $a$ and $q \rightarrow p q$.

If one takes an arbitrary ratio of elliptic gamma functions whose arguments are given by integer powers of the fugacities $v, z_{i}, x_{j}, y_{j}$, then the $q$-certificates will be again given by ratios of theta-functions. However, $p$-shifts of the fugacities in these certificates would produce in general arbitrary quasiperiodicity factors described by some powers of all fugacities (which are all equal to 1 in our case).

Equations (3.14) fix the second current to be again the gauge current since we are taking $p$-shifts for the $z_{j}$-variable and the resulting quasiperiodic factor phases will necessarily contain $m_{j}^{(a)}$-power. The third current in the triangle anomaly is fixed by considering in the resulting phase the powers of fugacities $v$ (for the $\mathrm{U}(1)_{B^{-}}$current), $x_{k}$ (for the $\mathrm{SU}\left(N_{f}\right)_{l^{-}}$ current), $y_{k}$ (for the $\mathrm{SU}\left(N_{f}\right)_{r}$-current) and for obtaining insertion of the $\mathrm{U}(1)_{R}$-current one should trace the powers $(p q)^{R / 2}$.

Let us pick up cubic products of $m_{i}^{a}$ (3.10) corresponding to the gauge group fugacities and sum over $a$ - this sum corresponds to the anomaly coefficient for $\left\langle j_{G}^{i, \mu} j_{G}^{j, \nu} j_{G}^{k, \lambda}\right\rangle$ with color indices $i, j, k$. It is easy to see that it vanishes, moreover, its pieces coming from the gluinos (i.e., from the terms $\propto \Gamma\left(z_{i} z_{j}^{-1}, z_{i}^{-1} z_{j}\right)$ and the chiral fields vanish independently. Cancellation of the powers of the $v$-variables in (3.14) tells that the gauge anomaly $\mathrm{SU}\left(N_{c}\right)^{2} \mathrm{U}(1)_{B}$ is absent, and similar situation holds for $\mathrm{SU}\left(N_{c}\right)^{2} \mathrm{SU}\left(N_{f}\right)_{l, r}$-anomalies.

If the $R$-charge is not fixed in advance, then there emerge quasiperiodicity multipliers given by some powers of $p q$, as indicated in (3.14). The demand of the absence of these multipliers fixes the $R$-charge in the same way as the vanishing of gauge anomaly $\left\langle j_{G}^{a \mu} j_{G}^{b \nu} j_{\mathrm{U}(1)_{R}}^{\rho}\right\rangle=0$ does, $N_{f}-N_{c}-R N_{f}=0$. Absence of the asymmetry in $p$ and $q$ in these multipliers, despite of such asymmetry present in (3.13), corresponds to the energymomentum conservation. 
Equations (3.15) correspond to the choice of the second current in the anomaly triangle diagram as $\mathrm{SU}\left(N_{f}\right)_{l, r}$-currents since we scale respective fugacities. Then the third current is determined from the quasiperiodicity factors. Absence of such factors in our case shows that all corresponding anomalies vanish. Thus, separate vanishing of polynomials (3.10)-(3.12) for electric and magnetic theories, when at least one of $m_{i}^{(a)}$-variables comes from gauge group fugacities, describes cancellation of gauge anomalies and various conservation laws.

One can consider in a similar way other certificates and interpret corresponding ellipticity constraints as anomaly matching conditions, but this construction is not that lucid and evident as one would want to. Moreover, since there is no separate fugacity for $\mathrm{U}(1)_{R}$-group, there is no $q$-certificate associated with this group which would correspond to the insertion of $\mathrm{U}(1)_{R^{-}}$-current alone. Therefore, it is not possible to describe $\mathrm{U}(1)_{R}$ and $\mathrm{U}(1)_{R}^{3}$ anomalies in this way. Similar conclusion has been reached recently by Sudano [36] following our considerations in [10, 30].

Let us show that the $\mathrm{SL}(3, \mathbb{Z})$-modular properties of elliptic hypergeometric terms produce all anomaly matching conditions at once in a very simple way.

\section{$4 \mathrm{SL}(3, \mathbb{Z})$-modularity and anomalies}

In [33] the modified versions of elliptic hypergeometric integrals have been introduced. They satisfy the general definition of elliptic hypergeometric integrals of [6] mentioned above, but they are built from the modified elliptic gamma functions. Consider modifications of integrals (1.4) and (1.7). For this introduce new parametrization of fugacities

$$
\begin{gathered}
z_{j}=e^{2 \pi \mathrm{i} u_{j} / \omega_{2}}, j=1, \ldots, N_{c}, \quad \widetilde{z}_{j}=e^{2 \pi \mathrm{i} v_{j} / \omega_{2}}, j=1, \ldots, \widetilde{N}_{c}, \\
s_{k}=e^{2 \pi \mathrm{i} \alpha_{k} / \omega_{2}}, \quad t_{k}=e^{2 \pi \mathrm{i} \beta_{k} / \omega_{2}}, k=1, \ldots, N_{f} .
\end{gathered}
$$

Define now the following integrals

$$
I_{E}^{\bmod }=\kappa_{N_{c}}^{\bmod } \int_{-\omega_{3} / 2}^{\omega_{3} / 2} \frac{\prod_{i=1}^{N_{f}} \prod_{j=1}^{N_{c}} \mathcal{G}\left(\alpha_{i}+u_{j},-\beta_{i}-u_{j} ; \omega\right)}{\prod_{1 \leq i<j \leq N_{c}} \mathcal{G}\left(u_{i}-u_{j},-u_{i}+u_{j} ; \omega\right)} \prod_{j=1}^{N_{c}-1} \frac{d u_{j}}{\omega_{3}},
$$

where $\sum_{j=1}^{N_{c}} u_{j}=0, \mathcal{G}(a, b ; \omega):=\mathcal{G}(a ; \omega) \mathcal{G}(b ; \omega)$, and

$$
\begin{aligned}
I_{M}^{\bmod }= & \kappa_{\widetilde{N}_{c}}^{\bmod } \prod_{1 \leq i, j \leq N_{f}} \mathcal{G}\left(\alpha_{i}-\beta_{j} ; \omega\right) \\
& \times \int_{-\omega_{3} / 2}^{\omega_{3} / 2} \frac{\prod_{i=1}^{N_{f}} \prod_{j=1}^{\widetilde{N}_{c}} \mathcal{G}\left(\alpha / \widetilde{N}_{c}-\alpha_{i}+v_{j},-\beta / \widetilde{N}_{c}+\beta_{i}-v_{j} ; \omega\right)}{\prod_{1 \leq i<j \leq \widetilde{N}_{c}} \mathcal{G}\left(v_{i}-v_{j},-v_{i}+v_{j} ; \omega\right)} \prod_{j=1}^{\widetilde{N}_{c}-1} \frac{d v_{j}}{\omega_{3}},
\end{aligned}
$$

where $\widetilde{N}_{c}=N_{f}-N_{c}$ and $\sum_{j=1}^{\widetilde{N}_{c}} v_{j}=0$. The integration in both cases goes along the straight line segment connecting points $-\omega_{3} / 2$ and $\omega_{3} / 2$. The balancing condition reads

$$
\alpha-\beta=\left(N_{f}-N_{c}\right) \sum_{k=1}^{3} \omega_{k}, \quad \alpha=\sum_{i=1}^{N_{f}} \alpha_{i}, \quad \beta=\sum_{i=1}^{N_{f}} \beta_{i} .
$$


Finally,

$$
\kappa_{N_{c}}^{\bmod }=\frac{\kappa(\omega)^{N_{c}-1}}{N_{c} !}, \quad \kappa(\omega)=-\frac{\omega_{3}}{\omega_{2}} \frac{(p ; p)_{\infty}(q ; q)_{\infty}(r ; r)_{\infty}}{(\tilde{q} ; \tilde{q})_{\infty}} .
$$

These integrals are obtained from (1.4) and (1.7) after replacement of $\Gamma(z ; p, q)$ with $z=e^{2 \pi \mathrm{i} u / \omega_{2}}$ by the function $\mathcal{G}(u ; \omega)$ and some modification of the integration measure. Since both elliptic gamma functions solve the key equation (2.5), the modified elliptic hypergeometric integrals satisfy the same finite difference equations in the shifts $u \rightarrow u+\omega_{1}$ as the standard integrals do (and therefore modified identities can be proved similarly to the standard ones). However, they remain well defined for $|q|=1$ in difference from integrals (1.4) and (1.7).

Theorem 2 Suppose that

$$
\Im\left(\alpha_{i} / \omega_{3}\right), \Im\left(\left(\alpha / \widetilde{N}_{c}-\alpha_{i}\right) / \omega_{3}\right)<0, \quad \Im\left(\beta_{i} / \omega_{3}\right), \Im\left(\left(\beta / \widetilde{N}_{c}-\beta_{i}\right) / \omega_{3}\right)>0 .
$$

Then $I_{E}^{\bmod }=I_{M}^{\bmod }$.

The simplest proof follows the same lines as in [33], where a similar identity has been established for modified elliptic hypergeometric integrals of type II on the $B C_{n}$-root system. Namely, one should substitute to (4.2), (4.3) the modular transformed form of the modified elliptic gamma function (2.8) and simplify the combination of $B_{3,3}$-Bernoulli polynomials in the exponential factors. After application of the modular transformation law for the Dedekind eta-function $(2.11)$ to infinite products $(p ; p)_{\infty},(q ; q)_{\infty},(r ; r)_{\infty}$ these multipliers cancel out completely. As a result the equality $I_{E}^{\bmod }=I_{M}^{\bmod }$ reduces to the equality $I_{E}=I_{M}$ with the variables $s_{j}, t_{j}, p$ and $q$ replaced by $e^{-2 \pi \mathrm{i} \alpha_{j} / \omega_{3}}, e^{-2 \pi \mathrm{i} \beta_{j} / \omega_{2}}, \tilde{p}$ and $\tilde{r}$, respectively. The constraints imposed on the variables $\alpha_{j}$ and $\beta_{j}$ convert to the restrictions needed for guaranteeing the equality of integrals.

Denote as $I_{E, M}\left(\underline{\alpha}, \underline{\beta} ; \omega_{1}, \omega_{2}, \omega_{3}\right)$ the SCIs $I_{E, M}(s, t ; p, q)$ with the change of parameters (4.1). Then Theorem 2 states that integrals (4.2) and (4.3) are proportional to $\mathrm{SL}(3, \mathbb{Z})$-modular transformations $\left(\omega_{1}, \omega_{2}, \omega_{3}\right) \rightarrow\left(\omega_{1},-\omega_{3}, \omega_{2}\right)$ of the original integrals

$$
I_{E}^{\bmod }=e^{\varphi_{e}} I_{E}\left(\underline{\alpha}, \underline{\beta} ; \omega_{1},-\omega_{3}, \omega_{2}\right), \quad I_{M}^{\bmod }=e^{\varphi_{m}} I_{M}\left(\underline{\alpha}, \underline{\beta} ; \omega_{1},-\omega_{3}, \omega_{2}\right),
$$

and $\varphi_{e}=\varphi_{m}$. The latter equality appears to be nothing else than the 't Hooft anomaly matching conditions! Let us prove this statement in the general setting.

Given arbitrary $\mathbb{Z}^{n} \rightarrow \mathbb{Z}$ maps with finite support $m_{j}^{(a)} \in \mathbb{Z}, j=1, \ldots, n, \epsilon\left(m^{(a)}\right)=$ $\epsilon\left(m_{1}^{(a)}, \ldots, m_{n}^{(a)}\right), a=1, \ldots, K$, some $r_{-} \in \mathbb{Z}$ and real numbers $R^{(a)} \in \mathbb{R}$, we define a meromorphic function of $x_{i} \in \mathbb{C}^{*}, i=1, \ldots, n$,

$$
\Delta\left(x_{1}, \ldots, x_{n} ; p, q\right)=(p ; p)_{\infty}^{r_{-}}(q ; q)_{\infty}^{r_{-}} \prod_{a=1}^{K} \Gamma\left((p q)^{\frac{R^{(a)}}{2}} x_{1}^{m_{1}^{(a)}} x_{2}^{m_{2}^{(a)}} \ldots x_{n}^{m_{n}^{(a)}} ; p, q\right)^{\epsilon\left(m^{(a)}\right)} .
$$

One can demand that the powers of $p q$ are not removable by the transformations $x_{j} \rightarrow$ $(p q)^{\gamma_{j}} x_{j}$, i.e. that there do not exist real numbers $\gamma_{j}$ such that $R^{(a)} / 2+\sum_{j=1}^{n} \gamma_{j} m_{j}^{(a)}=0$. However, we shall not require this for simplicity. 
Denote now $x_{j}=e^{2 \pi \mathrm{i} u_{j} / \omega_{2}}$ and introduce the following meromorphic function of $u_{j} \in \mathbb{C}$ :

$$
\Delta^{\bmod }\left(u_{1}, \ldots, u_{n} ; \omega\right)=\kappa(\omega)^{r_{-}} \prod_{a=1}^{K} \mathcal{G}\left(R^{(a)} \sum_{k=1}^{3} \frac{\omega_{k}}{2}+\sum_{j=1}^{n} u_{j} m_{j}^{(a)} ; \omega\right)^{\epsilon\left(m^{(a)}\right)}
$$

The modular transformation properties of the totally elliptic hypergeometric terms were investigated in [30]. In the present context we have the following theorem.

Theorem 3 The $\mathrm{SL}(3, \mathbb{Z})$-modular transformation invariance relation

$$
\Delta^{\bmod }\left(u_{1}, \ldots, u_{n} ; \omega\right)=\Delta\left(e^{-2 \pi \mathrm{i} u_{1} / \omega_{3}}, \ldots, e^{-2 \pi \mathrm{i} u_{n} / \omega_{3}} ; \tilde{p}, \tilde{r}\right)
$$

leads to the following set of equations

$$
\begin{aligned}
\sum_{a=1}^{K} \epsilon\left(m^{(a)}\right) m_{i}^{(a)} m_{j}^{(a)} m_{k}^{(a)} & =0 \\
\sum_{a=1}^{K} \epsilon\left(m^{(a)}\right) m_{i}^{(a)} m_{j}^{(a)}\left(R^{(a)}-1\right) & =0 \\
\sum_{a=1}^{K} \epsilon\left(m^{(a)}\right) m_{i}^{(a)}\left(R^{(a)}-1\right)^{2} & =0 \\
\sum_{a=1}^{K} \epsilon\left(m^{(a)}\right) m_{i}^{(a)} & =0 \\
\sum_{a=1}^{K} \epsilon\left(m^{(a)}\right)\left(R^{(a)}-1\right)^{3}+r_{-} & =0 \\
\sum_{a=1}^{K} \epsilon\left(m^{(a)}\right)\left(R^{(a)}-1\right)+r_{-} & =0 .
\end{aligned}
$$

The proof is simple enough. From representation (2.8) is it easy to see that

$$
\begin{aligned}
\frac{\Delta^{\mathrm{mod}}}{\Delta}=\frac{\kappa(\omega)^{r_{-}}}{(p ; p)_{\infty}^{r_{-}}(q ; q)_{\infty}^{r_{-}}} \prod_{a=1}^{K} \exp & {\left[-\frac{\pi \mathrm{i} \epsilon\left(m^{(a)}\right)}{3 \omega_{1} \omega_{2} \omega_{3}}\left(\frac{R^{(a)}-1}{2} \sum_{k=1}^{3} \omega_{k}+\sum_{i=1}^{n} u_{i} m_{i}^{(a)}\right)\right.} \\
\times & \left.\left(\left(\frac{R^{(a)}-1}{2} \sum_{k=1}^{3} \omega_{k}+\sum_{i=1}^{n} u_{i} m_{i}^{(a)}\right)^{2}-\frac{1}{4} \sum_{k=1}^{3} \omega_{k}^{2}\right)\right]=1 .
\end{aligned}
$$

Since chemical potentials $u_{i}$ are continuous independent variables, the polynomial in the exponential depending on them should vanish. Picking up the cubic terms $u_{i} u_{j} u_{k}$ one obtains equation (4.7), the quadratic terms yield (4.8), there are two terms linear in $u_{i}$ with the coefficients depending on continuous modular parameters $\omega_{k}$ in different way. Vanishing of these terms yields two equations (4.9) and (4.10). Finally, we are left with 
the equation

$$
\begin{aligned}
{\left[-\frac{\omega_{3}(p ; p)_{\infty}(q ; q)_{\infty}(r ; r)_{\infty}}{\omega_{2}(\tilde{p} ; \tilde{p})_{\infty}(\tilde{q} ; \tilde{q})_{\infty}(\tilde{r} ; \tilde{r})_{\infty}}\right]^{r_{-}} \prod_{a=1}^{K} \exp \left[-\frac{\pi \mathrm{i} \epsilon\left(m^{(a)}\right)}{24 \omega_{1} \omega_{2} \omega_{3}}\left(\sum_{k=1}^{3} \omega_{k}\right)\right.} & \\
\left.\times\left(R^{(a)}-1\right)\left(\left(R^{(a)}-1\right)^{2}\left(\sum_{k=1}^{3} \omega_{k}\right)^{2}-\sum_{k=1}^{3} \omega_{k}^{2}\right)\right] & =1 .
\end{aligned}
$$

Applying the modular transformation formula (2.11) to infinite products and using arbitrariness of variables $\omega_{k}$ we come to the last two equations (4.11) and (4.12).

Suppose now that the powers $(p q)^{R^{(a)} / 2}$ can be removed from (4.4) by redefinition of variables $x_{i} \rightarrow(p q)^{\gamma_{i}} x_{i}$, i.e. that there exist some numbers $\gamma_{i}$ such that $R^{(a)}=$ $-2 \sum_{i=1}^{n} \gamma_{i} m_{i}^{(a)}$. Substituting these conditions to (4.8), (4.9), we immediately see that they reduce to equations (3.11), (3.12), i.e. the situation becomes similar to the original Seiberg duality case. Interestingly, equations (4.11) and (4.12) are reduced in this case to one constraint

$$
\sum_{a=1}^{K} \epsilon\left(m^{(a)}\right)=r_{-} .
$$

If the ranks of dual gauge groups are equal (e.g., for self-dual theories), one has $\sum_{a=1}^{K} \epsilon\left(m^{(a)}\right)=0$. Equation (4.13) thus completes equations (3.10)-(3.12) to guarantee $\mathrm{SL}(3, \mathbb{Z})$-modular invariance of such elliptic hypergeometric terms [30].

It is evident that equations (4.7)-(4.12) coincide with the 't Hooft anomaly matching conditions for dual theories with the $\Delta$-function being built as the ratio of kernels of elliptic hypergeometric integrals describing electric and magnetic SCIs. We have checked this statement explicitly for the original Seiberg duality using the kernels of modified elliptic hypergeometric integrals (4.2) and (4.3) with the substitutions

$$
\begin{aligned}
& \alpha_{i}=R\left(\omega_{1}+\omega_{2}+\omega_{3}\right) / 2+\eta+\delta_{i}, \\
& \beta_{i}=-R\left(\omega_{1}+\omega_{2}+\omega_{3}\right) / 2+\eta+\xi_{i}, \quad i=1, \ldots, N_{f},
\end{aligned}
$$

where $\eta$ is the chemical potential for $\mathrm{U}(1)_{B}$-group, $\delta_{i}$ and $\xi_{i}$ are chemical potentials for $\mathrm{SU}\left(N_{f}\right)_{l}$ and $\mathrm{SU}\left(N_{f}\right)_{r}$ groups, $\sum_{i=1}^{N_{f}} \delta_{i}=\sum_{i=1}^{N_{f}} \xi_{i}=0$. The general rule of getting the anomaly coefficients is very simple: expand $\mathrm{SL}(3, \mathbb{Z})$-phase factor and associate the gauge and flavor group currents with the corresponding chemical potentials and the $\mathrm{U}(1)_{R^{-}}$-current with the term $R^{(a)}-1$, describing the $R$-charge of the chiral fermions and for $R^{(a)}=0$ modelling the contribution of gluinos. Since we have a cubic polynomial in these variables we model the triangle anomaly diagram. For instance, the plain chiral superfield contributes to the modular phase the term $\propto B_{3,3}\left(R\left(\omega_{1}+\omega_{2}+\omega_{3}\right) / 2 ; \omega\right)$ which is easily seen to contain only two pieces $\propto(R-1)^{3}$ and $\propto(R-1)$, as needed for $\mathrm{U}(1)_{R}^{3}$ and $\mathrm{U}(1)_{R}$-anomalies.

Computing the modular transformation exponential factors for the electric theory alone we explicitly see emergence of all anomaly coefficients (coinciding with the magnetic theory coefficients):

- Cubic polynomials depending on the integration variables $u_{i}$ or $v_{i}$ lead to equations (4.7)-(4.10) with at least one index $i$ coming from the gauge groups. They 
vanish separately in electric and magnetic theories (this is true for any duality, not just the Seiberg case) leading to $\left\langle j_{G}^{a, \mu} j_{1}^{\nu} j_{2}^{\lambda}\right\rangle=0$ for any conserved current $j_{1,2}^{\lambda}$ including the energy momentum tensor. E.g., from equation (4.8) one finds $R=\left(N_{f}-N_{c}\right) / N_{f}$.

- The terms $\propto \delta_{i} \delta_{j} \delta_{k}$ corresponding to (4.7) yield the $\mathrm{SU}\left(N_{f}\right)_{l}^{3}$-anomaly coefficient $\propto N_{c}$ (with a similar result for $\left.\mathrm{SU}\left(N_{f}\right)_{r}^{3}\right)$.

- The terms $\propto \delta_{i} \delta_{j} \eta$ corresponding to (4.7) give the $\mathrm{SU}\left(N_{f}\right)_{l}^{2} \mathrm{U}(1)_{B}$-anomaly coefficient $\propto N_{c}$.

- The terms $\propto \delta_{i} \delta_{j}(R-1)$ corresponding to (4.8) give the $\mathrm{SU}\left(N_{f}\right)_{l}^{2} \mathrm{U}(1)_{R^{-} \text {-anomaly }}$ coefficient $\propto N_{c}^{2} / N_{f}$.

- The terms $\propto \eta^{2}(R-1)$ corresponding to (4.8) give the $\mathrm{U}(1)_{B}^{2} \mathrm{U}(1)_{R^{-a n o m a l y} \text { coeffi- }}$ cient $\propto N_{c}^{2}$.

- The terms $\propto(R-1)^{2}$ corresponding to (4.9) are absent leading to vanishing $\mathrm{U}(1)_{B} \mathrm{U}(1)_{R}^{2}$ and $\mathrm{SU}\left(N_{f}\right)_{l, r} \mathrm{U}(1)_{R}^{2}$-anomalies.

- Linear terms in flavor chemical potentials are absent (i.e., equations (4.10) are satisfied separately for electric and magnetic theories), which means that there are no $\mathrm{U}(1)_{B}$ and $\mathrm{SU}\left(N_{f}\right)_{l, r}$-anomalies.

- The electric part of equation (4.11) yields precisely the $\mathrm{U}(1)_{R}^{3}$-anomaly coefficient $2 N_{f} N_{c}(R-1)^{3}+N_{c}\left(N_{c}-1\right)+\operatorname{rank} G_{c}=-2 N_{c}^{4} / N_{f}^{2}+N_{c}^{2}-1$.

- The electric part of equation (4.12) yields precisely the $\mathrm{U}(1)_{R^{-}}$-anomaly coefficient $2 N_{f} N_{c}(R-1)+N_{c}\left(N_{c}-1\right)+\operatorname{rank} G_{c}=-N_{c}^{2}-1$.

If $\Im\left(\omega_{1} / \omega_{2}\right)>0$ then one can take the limit $\omega_{3} \rightarrow \infty$ and obtain

$$
\lim _{\omega_{3} \rightarrow \infty} \mathcal{G}(u ; \omega)=\frac{\left(e^{2 \pi \mathrm{i} u / \omega_{1}} \widetilde{q} ; \widetilde{q}\right)_{\infty}}{\left(e^{2 \pi \mathrm{i} u / \omega_{2}} ; q\right)_{\infty}} .
$$

Taking this limit in the relation $I_{E}^{\mathrm{mod}}=I_{M}^{\bmod }$ one gets the equality of partition functions of some $3 d \mathcal{N}=2$ theories which is similar to the reduction of standard $4 d$ SCIs to $3 d$ partition functions [31]. The main difficulty in finding $3 d$ Seiberg dualities consists in the absence of the anomaly matching conditions. Starting from known $4 d$ dualities and using the limit (4.14) one automatically and easily derives $3 d$ dual theories which comprise (in a hidden way) $4 d$ anomaly matching conditions.

\section{Total ellipticity and modularity. The general case}

Let us consider the total ellipticity condition for general elliptic hypergeometric term (4.4). Corresponding $q$-certificates have the form

$$
h_{i}(x, q ; p)=\prod_{a=1}^{K} \prod_{l=0}^{m_{i}^{(a)}-1} \theta\left(q^{l}(p q)^{R^{(a)} / 2} \prod_{k=1}^{n} x_{k}^{m_{k}^{(a)}} ; p\right)^{\epsilon\left(m^{(a)}\right)} .
$$


Recursively using relation $(2.2)$ one can verify that the condition $h_{i}\left(\ldots p x_{j} \ldots, q ; p\right)=$ $h_{i}(x, q ; p)$ yields equations (4.7) and (4.8) together with the constraint

$$
\sum_{a=1}^{K} \epsilon\left(m^{(a)}\right) m_{i}^{(a)} m_{j}^{(a)} \in 2 \mathbb{Z}
$$

coming from the positive sign prescription.

In order to investigate $p$-periodicity properties of $h_{i}(x, q ; p)(5.1)$ it is necessary to introduce a new parameter $L$, a minimal positive integer such that all $L R^{(a)} \in 2 \mathbb{Z}$. Note that this requires an advance knowledge of some properties of $R$-charges, which are presumed to be fixed from the anomaly cancellation/matching conditions. Therefore the constraint $h_{i}\left(x, p^{L} q ; p\right)=h_{i}(x, q ; p)$ looks a little bit unnatural from the physical point of view. Nevertheless, it yields equations (4.9) and (4.10) together with the constraint

$$
L \sum_{a=1}^{K} \sum_{a=1}^{K} \epsilon\left(m^{(a)}\right) m_{i}^{(a)}\left(m_{i}^{(a)}+R^{(a)}\right) \in 4 \mathbb{Z}
$$

Equations (4.7)-(4.10) and the prescriptions (5.2), (5.3) were derived from the total ellipticity condition by the first author (unpublished) in a slightly different form during the work on [30] and more recently by Sudano in [36] (where one can find the details of computations).

On the one hand, both equations (5.2) and (5.3) do not emerge from the $\mathrm{SL}(3, \mathbb{Z})$ modular invariance condition (4.6). On the other hand, checks of the total ellipticity condition for all known dualities performed in [10] show that they are satisfied in physical theories. In some cases it can be shown that they follow from equations (4.7)-(4.12) (e.g., for $\left.R^{(a)} \propto \sum_{i=1}^{n} \gamma_{i} m_{i}^{(a)}\right)$. Therefore we conjecture that equations (5.2) and (5.3) are automatically satisfied for elliptic hypergeometric integrals associated with nontrivial electric-magnetic dualities. If it were true, one could state that the condition of total ellipticity of elliptic hypergeometric terms is necessary, but not sufficient for guaranteeing the 't Hooft anomaly matching conditions.

Finally, we have a generalization of Theorem 1.

Corollary 1 Suppose (4.4) is a totally elliptic hypergeometric term. Then all its qcertificates $h_{i}(x, q ; p)(5.1)$ are modular invariant.

For proving this statement consider the ratio of modular transformed certificates

$$
\frac{\tilde{h}_{i}}{h_{i}}=\prod_{a=1}^{K} \prod_{\ell=0}^{m_{i}^{(a)}-1} \frac{\theta\left(e^{-2 \pi \mathrm{i} \gamma_{\ell}^{a} / \omega_{3}} ; \tilde{p}\right)^{\epsilon\left(m^{(a)}\right)}}{\theta\left(e^{2 \pi \mathrm{i} \gamma_{\ell}^{a} / \omega_{2}} ; p\right)^{\epsilon\left(m^{(a)}\right)}}, \quad \gamma_{\ell}^{a}=R^{(a)} \sum_{k=1}^{3} \frac{\omega_{k}}{2}+\sum_{j=1}^{n} u_{j} m_{j}^{(a)}+\omega_{1} \ell
$$

Using the modular transformation law for theta functions (2.10) one easily checks that $\tilde{h}_{i} / h_{i}=1$ as a consequence of equations (4.7)-(4.10). 


\section{Conclusion}

In [10] we formulated the conjecture that all 't Hooft anomaly matching conditions follow from the total ellipticity condition [30]. It was based on the observation that some of the anomaly coefficients emerge from the nontrivial quasiperiodicity factors appearing during the checks of ellipticity of the certificates (5.1) (in particular, triviality of some factors meant the absence of gauge anomalies). However, we did not perform a systematic study of this relation at that time. Later in [31] we noticed importance of the $\mathrm{SL}(3, \mathbb{Z})$-modularity properties for this problem.

In this work we presented a systematic derivation of the triangle anomaly coefficients for general theories out of the $\mathrm{SL}(3, \mathbb{Z})$-group modular transformation properties of the kernels of dual indices. The generalized Rains-Spiridonov equations (4.7)-(4.12) are interpreted as the universal 't Hooft anomaly matching conditions for $4 d$ supersymmetric field theories. In particular, we explicitly checked emergence of all anomaly coefficients for the original Seiberg duality.

Still, the general physical meaning of the modular transformation properties of SCIs remains unknown. It is necessary to find physical derivation of the modified elliptic hypergeometric integrals as some kind of modified SCIs. Perhaps they are related to computing indices in $\mathcal{N}=1$ theories quantized on $\mathbb{T}^{3} \times \mathbb{R}$. In [37] $4 d \mathcal{N}=4 \mathrm{SYM}$ theories with simply laced gauge groups were considered on such a space-time. One can rewrite all SCIs, in particular, SCIs of $4 d \mathcal{N}=4 \mathrm{SYM}$ theories listed in [12], as some modified elliptic hypergeometric integrals and try to associate our $\mathrm{SL}(3, \mathbb{Z})$-modular transformations with the natural $\mathrm{SL}(3, \mathbb{Z})$-group action in the taken space-time.

Actually, we have demonstrated coincidence of anomaly matching conditions with some mathematical properties of SCIs, but we did not derive these conditions from first principles. A proper mathematical consideration of the problem should use the formal mathematical definition of anomalies as cocycles of gauge groups (see, e.g., [38, 39]) yielding anomaly matching condition as an equality of Chern classes of dual theories. This should yield also the proper cohomological meaning of the modular invariance condition for elliptic hypergeometric terms. From the physical side, it is necessary to compute the effect of SL $(3, \mathbb{Z})$-modular transformation on SCIs and demonstrate explicitly how the anomaly diagrams emerge in the corresponding changes of SCIs.

From the group-theoretical point of view the anomaly coefficients are described by certain combination of Casimir invariants. It seems possible to trace how these invariants emerge in the modular transformation phase using the group-theoretical information hidden in the definition of SCIs having the elliptic hypergeometric terms of a specific form (e.g., $r_{-}$is fixed from a piece of the characters of adjoint representations of gauge groups). This should also yield anomaly matching conditions.

We would like to finish by posing an interesting mathematical problem of describing universal restrictions on powers $m_{i}^{(a)}$ and $\epsilon\left(m^{(a)}\right)$ in the general elliptic hypergeometric term (4.4) which would force this term to become a ratio of two kernels of SCIs (1.3). Equations (4.7)-(4.12) are necessary for this, but not sufficient. Such constraints would provide a powerful mathematical tool for building physical dualities for supersymmetric field theories. 
Dedication. This paper is dedicated to the memory of our friend and collaborator Francis Dolan. We got acquainted with him because of his beautiful work with Hugh Osborn on the connection of superconformal indices with the elliptic hypergeometric integrals [1]. From November 2008 we were exchanging with him by many e-mails, discussed various aspects of this interrelation and had vast plans for joint work. Unfortunately, we were able to write only one joint paper [31]. We shall remember Francis as a good friend and a brilliant scientist with original ideas, and we miss him much.

\section{Acknowledgments}

This work is supported in part by RFBR grant no. 12-01-00242 and the Heisenberg-Landau program. The authors are indebted to H. Osborn and V. A. Rubakov for valuable discussions and helpful remarks. GV would like to thank BLTP, JINR in Dubna for hospitality in January 2012 during the workshop "Classical and Quantum Integrable Systems" where the results of this paper were presented.

Open Access. This article is distributed under the terms of the Creative Commons Attribution License which permits any use, distribution and reproduction in any medium, provided the original author(s) and source are credited.

\section{References}

[1] F. Dolan and H. Osborn, Applications of the superconformal index for protected operators and q-hypergeometric identities to $N=1$ dual theories, Nucl. Phys. B 818 (2009) 137 [arXiv: 0801.4947] [INSPIRE].

[2] J. Kinney, J.M. Maldacena, S. Minwalla and S. Raju, An index for 4 dimensional super conformal theories, Commun. Math. Phys. 275 (2007) 209 [hep-th/0510251] [INSPIRE].

[3] C. Römelsberger, Counting chiral primaries in $N=1, D=4$ superconformal field theories, Nucl. Phys. B 747 (2006) 329 [hep-th/0510060] [INSPIRE].

[4] C. Römelsberger, Calculating the superconformal index and Seiberg duality, arXiv:0707.3702 [INSPIRE].

[5] V.P. Spiridonov, On the elliptic beta function, Russ. Math. Surv. 56 (2001) 185 [Usp. Mat. Nauk 56 (2001) 181].

[6] V.P. Spiridonov, Theta hypergeometric integrals, Alg. i Anal. 15 (6) (2003) 161 [St. Petersburg Math. J. 15 (2004) 929] [math/0303205].

[7] V.P. Spiridonov, Essays on the theory of elliptic hypergeometric functions, Russ. Math. Surv. 63 (2008) 405 [Usp. Mat. Nauk 63 (2008) 3] [arXiv:0805.3135].

[8] N. Seiberg, Electric-magnetic duality in supersymmetric nonAbelian gauge theories, Nucl. Phys. B 435 (1995) 129 [hep-th/9411149] [INSPIRE].

[9] V. Spiridonov and G. Vartanov, Superconformal indices for $N=1$ theories with multiple duals, Nucl. Phys. B 824 (2010) 192 [arXiv:0811.1909] [INSPIRE].

[10] V. Spiridonov and G. Vartanov, Elliptic hypergeometry of supersymmetric dualities, Commun. Math. Phys. 304 (2011) 797 [arXiv:0910.5944] [INSPIRE]. 
[11] V. Spiridonov and G. Vartanov, Supersymmetric dualities beyond the conformal window, Phys. Rev. Lett. 105 (2010) 061603 [arXiv:1003.6109] [INSPIRE].

[12] V. Spiridonov and G. Vartanov, Superconformal indices of $\mathcal{N}=4$ SYM field theories, Lett. Math. Phys. 100 (2012) 97 [arXiv:1005.4196] [INSPIRE].

[13] G. Vartanov, On the ISS model of dynamical SUSY breaking, Phys. Lett. B 696 (2011) 288 [arXiv: 1009.2153] [INSPIRE].

[14] V. Spiridonov, Elliptic beta integrals and solvable models of statistical mechanics, Contemp. Math. 563 (2012) 181 [arXiv: 1011. 3798] [INSPIRE].

[15] V. Spiridonov and G. Vartanov, Elliptic hypergeometry of supersymmetric dualities II. Orthogonal groups, knots and vortices, arXiv:1107.5788 [INSPIRE].

[16] D. Sen, Supersymmetry in the space-time $R \times S^{3}$, Nucl. Phys. B 284 (1987) 201 [inSPIRE].

[17] G. Festuccia and N. Seiberg, Rigid supersymmetric theories in curved superspace, JHEP 06 (2011) 114 [arXiv:1105.0689] [INSPIRE].

[18] F.A. Dolan, e-mail message to the authors with unpublished notes on localization in $\mathcal{N}=1$ theories on $S^{3} \times \mathbb{R}$ (10 April 2011).

[19] A. Gadde, E. Pomoni, L. Rastelli and S.S. Razamat, S-duality and $2 d$ topological QFT, JHEP 03 (2010) 032 [arXiv:0910.2225] [INSPIRE].

[20] A. Gadde, L. Rastelli, S.S. Razamat and W. Yan, The superconformal index of the $E_{6} S C F T$, JHEP 08 (2010) 107 [arXiv: 1003.4244] [INSPIRE].

[21] A. Gadde, L. Rastelli, S.S. Razamat and W. Yan, The $4 D$ superconformal index from q-deformed 2d Yang-Mills, Phys. Rev. Lett. 106 (2011) 241602 [arXiv:1104.3850] [INSPIRE].

[22] A. Gadde, L. Rastelli, S.S. Razamat and W. Yan, Gauge theories and macdonald polynomials, arXiv:1110.3740 [INSPIRE].

[23] B.I. Zwiebel, Charging the superconformal index, JHEP 01 (2012) 116 [arXiv:1111.1773] [INSPIRE].

[24] F. Benini, T. Nishioka and M. Yamazaki, $4 D$ Index to $3 D$ index and $2 d T Q F T$, arXiv: 1109.0283 [INSPIRE].

[25] Y. Nakayama, $4 D$ and $2 D$ superconformal index with surface operator, JHEP 08 (2011) 084 [arXiv:1105.4883] [INSPIRE].

[26] T. Dimofte, D. Gaiotto and S. Gukov, 3-manifolds and 3D indices, arXiv:1112.5179 [INSPIRE].

[27] D. Gang, E. Koh and K. Lee, Line operator index on $S^{1} \times S^{3}$, arXiv:1201.5539 [INSPIRE].

[28] E.M. Rains, Transformations of elliptic hypergeometric integrals, Ann. of Math. 171 (2010) 169.

[29] G. 't Hooft, Naturalness, chiral symmetry, and spontaneous chiral symmetry breaking, Recent developments in gauge theories G. 't Hooft et al. eds., Plenum Press, New York U.S.A. (1980).

[30] V. Spiridonov, Elliptic hypergeometric terms, SMF Séminaire et Congrès 23 (2011) 385 [arXiv: 1003.4491] [INSPIRE].

[31] F. Dolan, V. Spiridonov and G. Vartanov, From $4 D$ superconformal indices to $3 D$ partition functions, Phys. Lett. B 704 (2011) 234 [arXiv:1104.1787] [INSPIRE]. 
[32] G. Felder and A. Varchenko, The elliptic gamma function and $\mathrm{SL}(3, \mathbb{Z}) \ltimes \mathbb{Z}^{3}$, Adv. Math. 156 (2000) 44.

[33] J.F. van Diejen and V.P. Spiridonov, Unit circle elliptic beta integrals, Ramanujan J. 10 (2005) 187 [math/0309279].

[34] J.H. Brodie, P.L. Cho and K.A. Intriligator, Misleading anomaly matchings?, Phys. Lett. B 429 (1998) 319 [hep-th/9802092] [INSPIRE].

[35] D. Kutasov and A. Schwimmer, On duality in supersymmetric Yang-Mills theory, Phys. Lett. B 354 (1995) 315 [hep-th/9505004] [INSPIRE].

[36] M. Sudano, The Romelsberger index, Berkooz deconfinement and infinite families of Seiberg duals, JHEP 05 (2012) 051 [arXiv: 1112.2996] [INSPIRE].

[37] M. Henningson and N. Wyllard, Low-energy spectrum of $N=4$ super-Yang-Mills on $T^{3}$ : fat connections, bound states at threshold and S-duality, JHEP 06 (2007) 001 [hep-th/0703172] [INSPIRE].

[38] A.G. Reiman, M.A. Semenov-Tian-Shansky and L.D. Faddeev, Quantum anomalies and cocycles on gauge groups, Funkt. Analiz i ego Pril. 18 (1984) 64 [Funct. Analysis and its Appl. 18 (1984) 319].

[39] J.A. Harvey, TASI 2003 lectures on anomalies, hep-th/0509097 [INSPIRE]. 